\title{
Impulsive Perturbations of a Three-Species Food Chain System with the Beddington-DeAngelis Functional Response
}

\author{
Younghae Do, ${ }^{1}$ Hunki Baek, ${ }^{2}$ and Dongseok Kim ${ }^{3}$ \\ ${ }^{1}$ Department of Mathematics, Kyungpook National University, Daegu 702-701, Republic of Korea \\ ${ }^{2}$ Department of Mathematics Education, Catholic University of Daegu, \\ Gyeongsan 712-702, Republic of Korea \\ ${ }^{3}$ Department of Mathematics, Kyonggi University, Suwon 443-760, Republic of Korea
}

Correspondence should be addressed to Hunki Baek, hkbaek@cu.ac.kr

Received 26 July 2011; Accepted 2 October 2011

Academic Editor: Elena Braverman

Copyright (c) 2012 Younghae Do et al. This is an open access article distributed under the Creative Commons Attribution License, which permits unrestricted use, distribution, and reproduction in any medium, provided the original work is properly cited.

The dynamics of an impulsively controlled three-species food chain system with the BeddingtonDeAngelis functional response are investigated using the Floquet theory and a comparison method. In the system, three species are prey, mid-predator, and top-predator. Under an integrated control strategy in sense of biological and chemical controls, the condition for extinction of the prey and the mid-predator is investigated. In addition, the condition for extinction of only the midpredator is examined. We provide numerical simulations to substantiate the theoretical results.

\section{Introduction}

Classical two-species continuous time systems such as a Lotka-Volterra system have been used to investigate the interaction between ecological populations. However, in order to understand a complex ecological system, it is necessary to study multispecies systems. For this reason, in this paper, we study three-species food chain system which appears when a toppredator feeds on a mid-predator, which in turn feeds a prey, specially assuming BeddingtonDeAngelis functional responses between species [1].

In recent decades, the effects of impulsive perturbations on population systems have been widely studied and discussed by a number of researchers [2-19]. Thus, in order to control an ecological environment, a discrete impulsive strategy has been suggested. Especially for the three-species food chain system, two impulsive control methods, biological and chemical controls, have been taken into account. Here, a biological control means impulsive and periodic releasing of top-predator to control lower-level populations and 
a chemical control means that as a result of spreading pesticide the population of all threespecies are impulsively lessened.

In this context, the impulsively controlled three-species food chain system with Beddington-DeAngelis functional responses was proposed and studied by Wang et al. [11] and their system can be described as the following impulsively perturbed system:

$$
\begin{aligned}
& \frac{d x(t)}{d t}=x(t)(a-b x(t))-\frac{c_{1} x(t) y(t)}{\alpha_{1}+x(t)+\beta_{1} y(t)} \\
& \frac{d y(t)}{d t}=\frac{k_{1} c_{1} x(t) y(t)}{\alpha_{1}+x(t)+\beta_{1} y(t)}-\frac{c_{2} y(t) z(t)}{\alpha_{2}+y(t)+\beta_{2} z(t)}-d_{1} y(t), \quad t \neq(n+l-1) T, t \neq n T, \\
& \frac{d z(t)}{d t}=\frac{k_{2} c_{2} y(t) z(t)}{\alpha_{2}+y(t)+\beta_{2} z(t)}-d_{2} z(t), \\
& \Delta x(t)=-\delta_{1} x(t), \\
& \Delta y(t)=-\delta_{2} y(t), \quad t=(n+l-1) T \\
& \Delta z(t)=-\delta_{3} z(t), \\
& \Delta x(t)=0, \\
& \Delta y(t)=0, \quad t=n T \\
& \Delta z(t)=p, \\
& \left(x\left(0^{+}\right), y\left(0^{+}\right), z\left(0^{+}\right)\right)=\left(x_{0}, y_{0}, z_{0}\right),
\end{aligned}
$$

where $x(t), y(\mathrm{t})$, and $z(t)$ are the densities of the lowest-level prey, mid-level predator, and top-predator at time $t$, respectively. In this system, the prey grows according to a logistic growth with an intrinsic growth rate $a$ and a carrying capacity $a / b$ incorporating the Beddington-DeAngelis functional response. For parameters settings, $k_{i}(i=1,2)$ are the conversion efficiencies, $d_{i}(i=1,2)$ are the mortality rates of the mid-level predator and the top-predator, $c_{i}(i=1,2)$ are the maximum numbers of preys that can be eaten by a predator per unit of time, $\alpha_{i}(i=1,2)$ are the saturation constants, and $\beta_{i}(i=1,2)$ scale the impact of the predator interference. For an impulsive control strategy, top-predators are impulsively released in the periodic fashion of the period $T$ by artificial breeding of species, in a fixed number $(p>0)$ at each time, and by introducing an impulsive catching or poisoning of the prey populations, fixed proportions $\delta_{1}, \delta_{2}, \delta_{3}$ of the prey, mid-level predator, and top predator are degraded in an impulsive and periodic fashion, with the same period, but at different moments. Here, all parameters except $l$ and $\delta_{i}(i=1,2,3)$ are positive, $0 \leq l<1$, $\Delta w(t)=w\left(t^{+}\right)-w(t)$ for $w \in\{x, y, z\}$, and $0 \leq \delta_{1}, \delta_{2}, \delta_{3}<1$.

Although the authors in [11] had introduced the important system (1.1) in a sense of impulsive controlling the food chain system, we find that there are many problems in their theoretical results, where they had showed rich dynamical behaviors in the numerical simulations including a quasiperiodic oscillation, narrow periodic widow, wide periodic window, chaotic band, and period doubling bifurcation, symmetry-breaking pitchfork bifurcation, period-halving bifurcation and crises [20-22].

The authors in [11] had argued that (1) the prey and mid-predator free periodic solution $\left(0,0, z^{*}(t)\right)$ is always unstable without having any condition and (2) the midpredator free periodic solution for the system is $\left(a / b, 0, z^{*}(t)\right)$. But, based on our theoretical computation, the periodic solution $\left(a / b, 0, z^{*}(t)\right)$ can be found only when $\delta_{1}=0$. It means that 
under an impulsive control of the population system, the solution $\left(a / b, 0, z^{*}(t)\right)$ is useless and nonmeaningful. In Section 2, we will give a general form $\left(x^{*}(t), 0, z^{*}(t)\right)$ of the mid-predator free solution. In the case that the prey is impulsively strong poisoned or caught, there is a possibility that the prey will be eradicated. To examine this possibility, we reinvestigate their system (1.1). Finally, we find out that their theoretical results shown in [11] are wrong. In this paper we thus may correct and rebuild their theoretical results, in particular, the conditions for stabilities of the periodic solutions $\left(0,0, z^{*}(t)\right)$ and the new mid-predator free periodic solution $\left(x^{*}(t), 0, \mathrm{z}^{*}(t)\right)$.

The main purpose of this paper is to reestablish the local and global stability for two periodic solutions $\left(0,0, z^{*}(t)\right)$ and $\left(x^{*}(t), 0, z^{*}(t)\right)$. In addition, we exhibit some numerical examples. To do it, this paper is organized as follows. In Section 2, we first review notations and theorems. Main theorems for two impulsive periodic solutions are given in Section 3. The mathematical proofs for our main results will be provided in Section 4 . Conclusions are presented in Section 5.

\section{Basic Strategy}

In this section we will consider definitions, notations, and auxiliary results for impulsively perturbed dynamical systems.

\subsection{Preliminaries}

Let us denote $\mathbb{N}$ by the set of all nonnegative integers, $\mathbb{R}_{+}=[0, \infty), \mathbb{R}_{+}^{*}=(0, \infty), \mathbb{R}_{+}^{3}=\{\mathbf{x}=$ $\left.(x(t), y(t), z(t)) \in \mathbb{R}^{3}: x(t), y(t), z(t) \geq 0\right\}$, and $f=\left(f_{1}, f_{2}, f_{3}\right)^{T}$ the mapping defined by the right-hand sides of the first three equations in (1.1).

Let $V: \mathbb{R}_{+} \times \mathbb{R}_{+}^{3} \rightarrow \mathbb{R}_{+}$, then $V$ is said to belong to class $V_{0}$ if

(1) $V$ is continuous on $((n-1) T,(n+l-1) T] \times \mathbb{R}_{+}^{3} \cup((n+l-1) T, n T] \times \mathbb{R}_{+}^{3}$ for each $\mathbf{x} \in \mathbb{R}_{+}^{3}, n \in \mathbb{N}$, and two limits $\lim _{(t, \mathbf{y}) \rightarrow\left((n+l-1) T^{+}, \mathbf{x}\right)} V(t, \mathbf{y})=V\left((n+l-1) T^{+}, \mathbf{x}\right)$ and $\lim _{(t, \mathbf{y}) \rightarrow\left(n T^{+}, \mathbf{x}\right)} V(t, \mathbf{y})=V\left(n T^{+}, \mathbf{x}\right)$ exist;

(2) $V$ is locally Lipschitzian in $\mathbf{x}$.

Definition 2.1. For $V \in V_{0}$, one defines the upper right Dini derivative of $V$ with respect to the impulsive differential system $(1.1)$ at $(t, \mathbf{x}) \in((n-1) T,(n+l-1) T] \times \mathbb{R}_{+}^{3} \cup((n+l-1) T, n T] \times \mathbb{R}_{+}^{3}$ by

$$
D^{+} V(t, \mathbf{x})=\limsup _{h \rightarrow 0+} \frac{1}{h}[V(t+h, \mathbf{x}+h f(t, \mathbf{x}))-V(t, \mathbf{x})]
$$

We suppose that $g: \mathbb{R}_{+} \times \mathbb{R}_{+} \rightarrow \mathbb{R}$ satisfies the following hypotheses: $(\mathrm{H}) g$ is continuous on $((n-1) T,(n+l-1) T] \times \mathbb{R}_{+}^{3} \cup((n+l-1) T, n T] \times \mathbb{R}_{+}^{3}$ and the limits $\lim _{(t, y) \rightarrow\left((n+l-1) T^{+}, x\right)} g(t, y)=g\left((n+l-1) T^{+}, x\right)$ and $\lim _{(t, y) \rightarrow\left(n T^{+}, x\right)} g(t, y)=g\left(n T^{+}, x\right)$ exist and are finite for $x \in \mathbb{R}_{+}$and $n \in \mathbb{N}$. 
Lemma 2.2 (see [23]). Suppose $V \in V_{0}$ and

$$
\begin{gathered}
D^{+} V(t, \mathbf{x}) \leq g(t, V(t, \mathbf{x})), \quad t \neq(n+l-1) T, \quad t \neq n T, \\
V\left(t, \mathbf{x}\left(t^{+}\right)\right) \leq \psi_{n}^{1}(V(t, \mathbf{x})), \quad t=(n+l-1) T, \\
V\left(t, \mathbf{x}\left(t^{+}\right)\right) \leq \psi_{n}^{2}(V(t, \mathbf{x})), \quad t=n T,
\end{gathered}
$$

where $g: \mathbb{R}_{+} \times \mathbb{R}_{+} \rightarrow \mathbb{R}$ satisfies $(H)$ and $\psi_{n}^{1}, \psi_{n}^{2}: \mathbb{R}_{+} \rightarrow \mathbb{R}_{+}$are nondecreasing for all $n \in \mathbb{N}$. Let $r(t)$ be the maximal solution for the impulsive Cauchy problem

$$
\begin{gathered}
u^{\prime}(t)=g(t, u(t)), \quad t \neq(n+l-1) T, \quad t \neq n T, \\
u\left(t^{+}\right)=\psi_{n}^{1}(u(t)), \quad t=(n+l-1) T, \\
u\left(t^{+}\right)=\psi_{n}^{2}(u(t)), \quad t=n T, \\
u\left(0^{+}\right)=u_{0},
\end{gathered}
$$

defined on $[0, \infty)$. Then $V\left(0^{+}, \mathbf{x}_{0}\right) \leq u_{0}$ implies that $V(t, \mathbf{x}(t)) \leq r(t), t \geq 0$, where $\mathbf{x}(t)$ is any solution of (2.2).

A similar result can be obtained when all conditions of the inequalities in the Lemma 2.2 are reversed. Using Lemma 2.2, it is easy to prove that the positive octant $\left(\mathbb{R}_{+}^{*}\right)^{3}$ is an invariant region for the system (1.1) (see Lemma 2.1 in [11]).

\subsection{Periodic Solutions}

In the case in which $y=0$, that is, mid-predator is eradicated, the system (1.1) is decoupled and led to two impulsive differential equations (2.4) and (2.6). Let us consider the properties of these impulsive differential equations. The following equation or a subsystem of system (1.1) is a periodically forced system:

$$
\begin{gathered}
x^{\prime}(t)=x(t)(a-b x(t)), \quad t \neq(n+l-1) T, \quad t \neq n T, \\
x\left(t^{+}\right)=\left(1-\delta_{1}\right) x(t), \quad t=(n+l-1) T, \\
x\left(t^{+}\right)=x(t), \quad t=n T, \\
x\left(0^{+}\right)=x_{0} .
\end{gathered}
$$

Straightforward computation for getting a positive periodic solution $x^{*}(t)$ of (2.4) yields the analytic form of $x^{*}(t)$ :

$$
x^{*}(t)=\frac{a \eta_{1} \exp (a t-\lambda)}{b\left[1-\eta_{1}+\eta_{1} \exp (a t-\lambda)\right]}, \quad(n+l-1) T<t \leq(n+l) T,
$$

where $\lambda=a(n+l-1) T$ and $\eta_{1}=\left(\left(1-\delta_{1}\right) \exp (a T)-1\right) /(\exp (a T)-1)$. 
In case that $\delta_{1}=0$, the system (2.4) is the general logistic equation. From the analytic solution form (2.5) we get that $\eta_{1}$ should be 1 . It implies that $x^{*}(t)=a / b$.

Lemma 2.3 (see [4]). The following statements hold.

(1) If $a T+\ln \left(1-\delta_{1}\right)>0$, then $\lim _{t \rightarrow \infty}\left|x(t)-x^{*}(t)\right|=0$ for all solutions $x(t)$ of (2.4) starting with $x_{0}>0$.

(2) If aT $+\ln \left(1-\delta_{1}\right) \leq 0$, then $x(t) \rightarrow 0$ as $t \rightarrow \infty$ for all solutions $x(t)$ of (2.4).

Next, we consider the impulsive differential equation as follows:

$$
\begin{gathered}
z^{\prime}(t)=-d_{2} z(t), \quad t \neq n T, \quad t \neq(n+l-1) T \\
z\left(t^{+}\right)=\left(1-\delta_{3}\right) z(t), \quad t=(n+l-1) T \\
z\left(t^{+}\right)=z(t)+p, \quad t=n T \\
z\left(0^{+}\right)=z_{0} .
\end{gathered}
$$

The system (2.6) is a periodically forced linear system and its positive periodic solution $z^{*}(t)$ will be obtained:

$$
\begin{aligned}
& z^{*}(t)= \begin{cases}\frac{p \exp \left[-d_{2}(t-(n-1) T)\right]}{1-\left(1-\delta_{3}\right) \exp \left(-d_{2} T\right)}, & (n-1) T<t \leq(n+l-1) T, \\
\frac{p\left(1-\delta_{3}\right) \exp \left[-d_{2}(t-(n-1) T)\right]}{1-\left(1-\delta_{3}\right) \exp \left(-d_{2} T\right)}, & (n+l-1) T<t \leq n T,\end{cases} \\
& z^{*}\left(0^{+}\right)=z^{*}\left(n T^{+}\right)=\frac{p}{1-\left(1-\delta_{3}\right) \exp \left(-d_{2} T\right)}, \\
& z^{*}\left((n+l-1) T^{+}\right)=\frac{p\left(1-\delta_{3}\right) \exp \left(-d_{2} l T\right)}{1-\left(1-\delta_{3}\right) \exp \left(-d_{2} T\right)} \text {. }
\end{aligned}
$$

Moreover, we may get that

$$
z(t)= \begin{cases}\left(1-\delta_{3}\right)^{n-1} z z+z^{*}(t), & (n-1) T<t \leq(n+l-1) T, \\ \left(1-\delta_{3}\right)^{n} z z+z^{*}(t), & (n+l-1) T<t \leq n T,\end{cases}
$$

is a solution of (2.6), where $z z=\left(z\left(0^{+}\right)-\left(p\left(1-\delta_{3}\right) e^{-T} /\left(1-\left(1-\delta_{3}\right) \exp \left(-d_{2} T\right)\right)\right)\right) \exp \left(-d_{2} t\right)$.

From (2.7) and (2.9), we thus get the following result.

Lemma 2.4. For every solution $z(t)$ and every positive periodic solution $z^{*}(t)$ of $(2.6)$, it follows that $z(t)$ tends to $z^{*}(t)$ as $t \rightarrow \infty$.

\section{Main Results}

In this section we study the local and global stability of the lowest-level prey and midlevel predator free periodic solution $\left(0,0, z^{*}(t)\right)$ and of the mid-level predator free periodic 
solution $\left(x^{*}(t), 0, z^{*}(t)\right)$. The authors in [11] had claimed that the solution $\left(0,0, z^{*}(t)\right)$ of the impulsive controlled system (1.1) is always unstable. In the biological point of view, this result is suspected in the sense that the prey and mid-predator will be eradicated under enough impulsive control term, especially, $\delta_{1}$. In Section 3.1 we will reinvestigate the stability of the periodic solution $\left(0,0, z^{*}(t)\right)$ and correct the misleading results shown in [11], and then the stability of the mid-level predator free periodic solution $\left(x^{*}(t), 0, z^{*}(t)\right)$ will be studied in Section 3.2.

\subsection{A Stability of a Periodic Solution with Prey and Mid-Predator Eradication}

We theoretically and numerically consider the stability of the periodic solution $\left(0,0, z^{*}(t)\right)$ with prey and mid-predator eradications.

Theorem 3.1. The periodic solution $\left(0,0, z^{*}(t)\right)$ is unstable if $a T+\ln \left(1-\delta_{1}\right)>0$, and globally asymptotically stable if aT $+\ln \left(1-\delta_{1}\right)<0$.

The above Theorem 3.1 says that in case that $\delta_{1}$ is sufficiently close to 1 to make $a T+$ $\ln \left(1-\delta_{1}\right)$ negative, the pesticide has a negative effect on the growth of prey in a certain period $T$. To set up a control strategy for impulsive systems, we have to consider the relationship between two important factors, that is, the natural growth rate and (chemical) controlled rate in a controlling period.

The proof of this theorem will be provided in Section 4, and we may numerically consider the dynamical feature related to Theorem 3.1. To do it, we first fix the parameters: $b=0.5, c_{1}=2, c_{2}=2, \alpha_{1}=0.1, \alpha_{2}=0.5, \beta_{1}=1, \beta_{2}=0.1, d_{1}=0.1, d_{2}=0.9, k_{1}=0.7, k_{2}=0.5, \delta_{2}=$ $0.0001, \delta_{3}=0.03, l=0.6, p=2.9$. If we choose the parameter $a=1, T=1, \delta_{1}=0.7$, then the value $a T+\ln \left(1-\delta_{1}\right) \approx-0.3$ is negative. It implies that trajectories asymptotically approach the periodic orbit $\left(0,0, z^{*}(t)\right)$ as shown in Figure 1. But, for different parameters setting having a positive value $a T+\ln \left(1-\delta_{1}\right)>0$, we may expect that a typical trajectory with an initial condition near $\left(0,0, z^{*}(t)\right)$ is repelled from the periodic solution $\left(0,0, z^{*}(t)\right)$. For instance, we choose the parameters $a=5, T=8, \delta_{1}=0.2$ and then $a T+\ln \left(1-\delta_{1}\right) \approx 39.77$. A repelling behavior of a trajectory with a starting point near $\left(0,0, z^{*}(t)\right)$ is shown in Figure 2 . It shows the instability of the prey and mid-predator free solution.

As shown in Figures 1 and 2, the dynamical behavior of the periodic solution $\left(0,0, z^{*}(t)\right)$ is depending on the stability condition, the positiveness or negativeness of the value $a T+\ln \left(1-\delta_{1}\right)$. This stability condition is related to the total growth $a T$ in a period $T$ and the term $\ln \left(1-\delta_{1}\right)$ representing the loss of the prey due to the impulsive control $\delta_{1}$ on the prey. It means that species will be eradicated or grow depending on the sum of a natural growth and an artificial loss (impulsive control).

\subsection{A Stability of a Periodic Solution with Mid-Predator Eradication}

In this section the stability of the periodic solution $\left(x^{*}(t), 0, z^{*}(t)\right)$ with mid-predator eradication will be considered. In Theorem 3.2, we will mention the conditions for local and global stability of the periodic orbit $\left(x^{*}(t), 0, z^{*}(t)\right)$. Compared to Theorem 3.1 , the positiveness of the value $a T+\ln \left(1-\delta_{1}\right)$ should be added in the condition for being the stable periodic orbit $\left(x^{*}(t), 0, z^{*}(t)\right)$. 


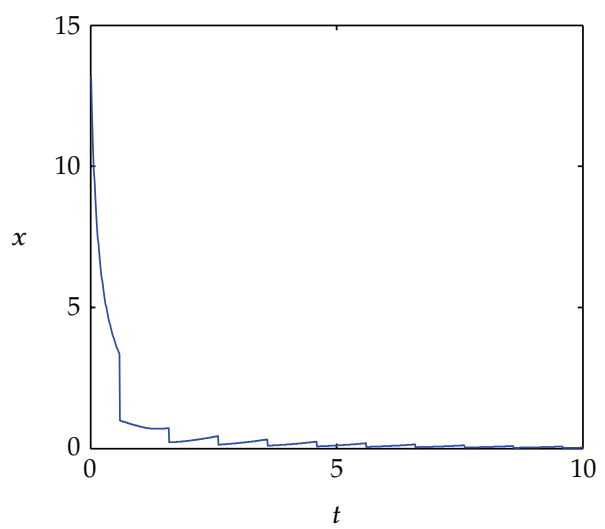

(a)

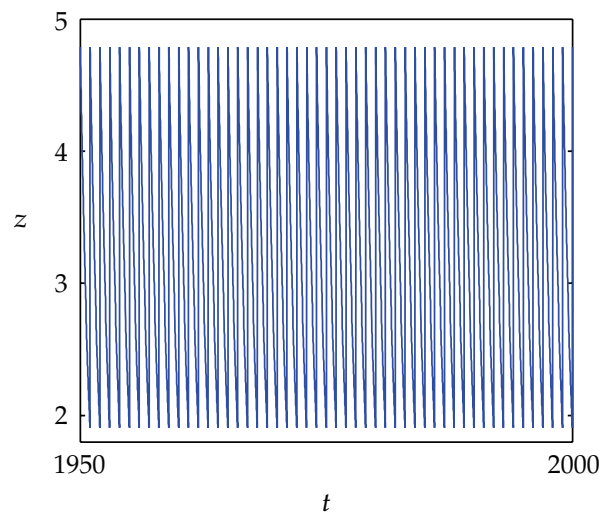

(c)

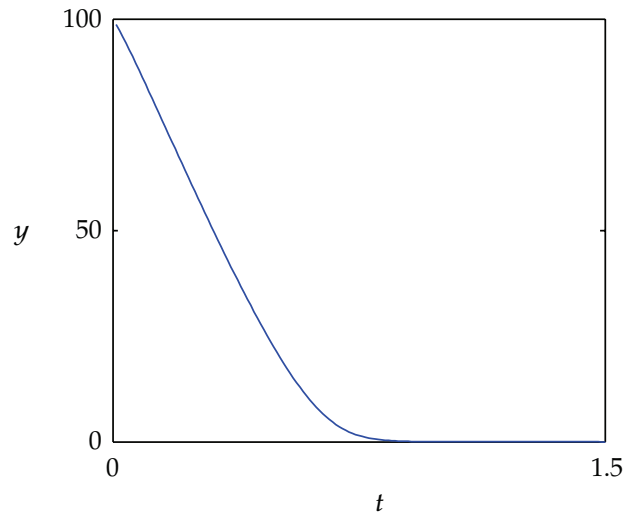

(e)

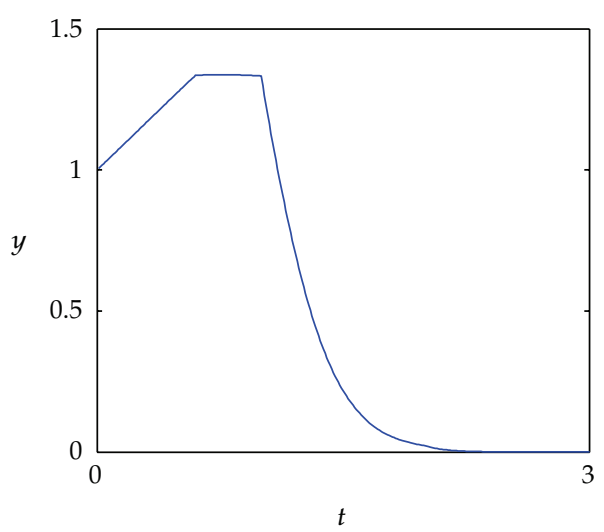

(b)

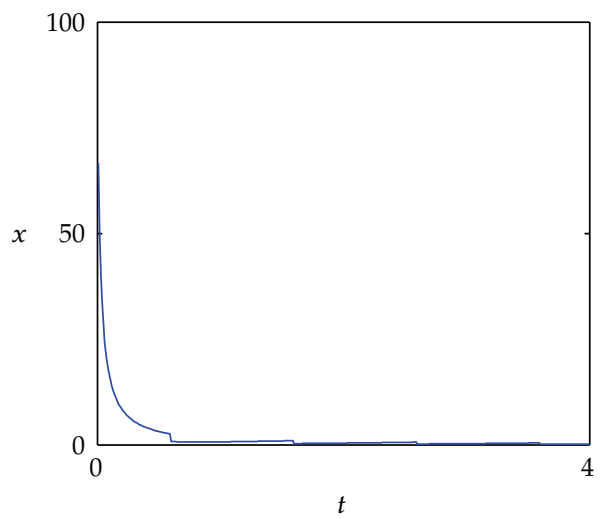

(d)

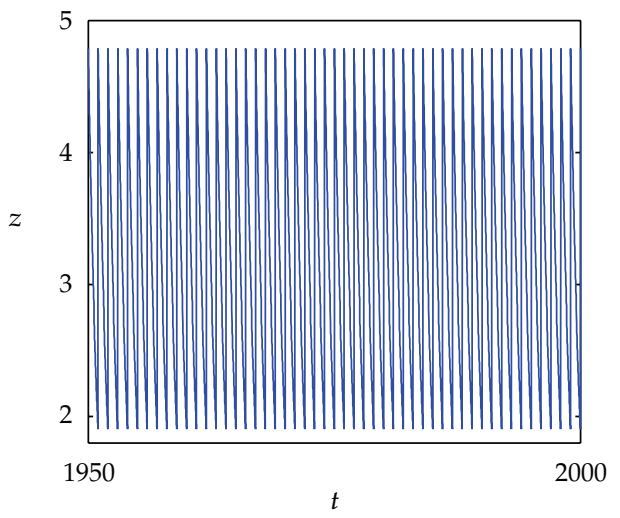

(f)

Figure 1: The dynamical behavior of the system (1.1) with parameters $a=1, T=1, \delta_{1}=0.7$. (a) $-(\mathrm{c})$ show that a trajectory with a starting point $\left(x_{0}, y_{0}, z_{0}\right)=(10,1,0.4)$ approaches to the periodic orbit $\left(0,0, z^{*}(t)\right)$. In (d)-(f) the behavior of trajectory with a different starting point $\left(x_{0}, y_{0}, z_{0}\right)=(100,100,100)$ is shown. 


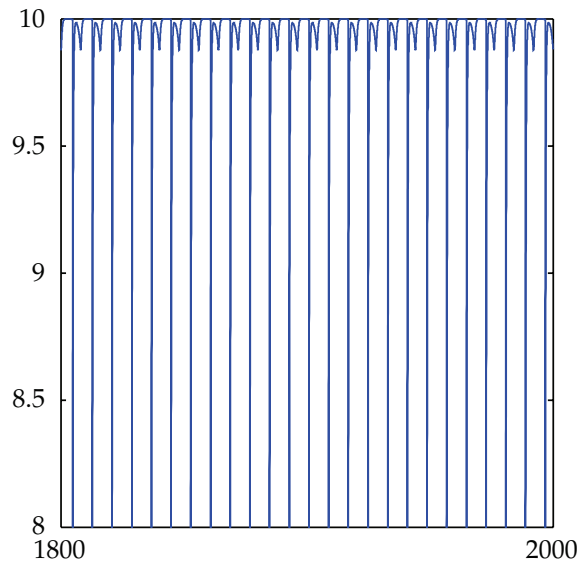

(a)

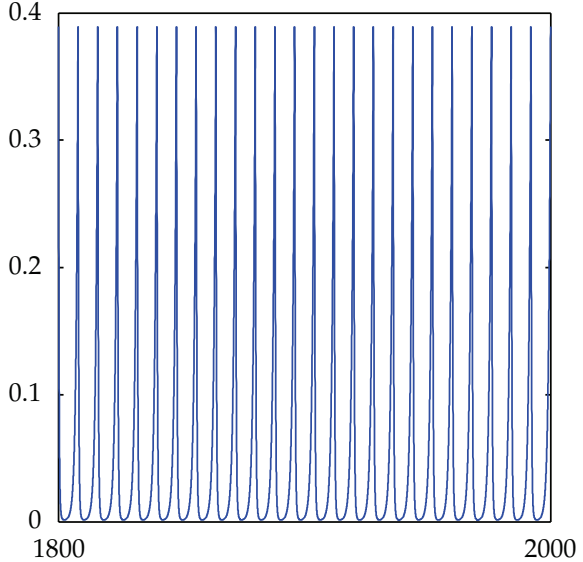

(b)

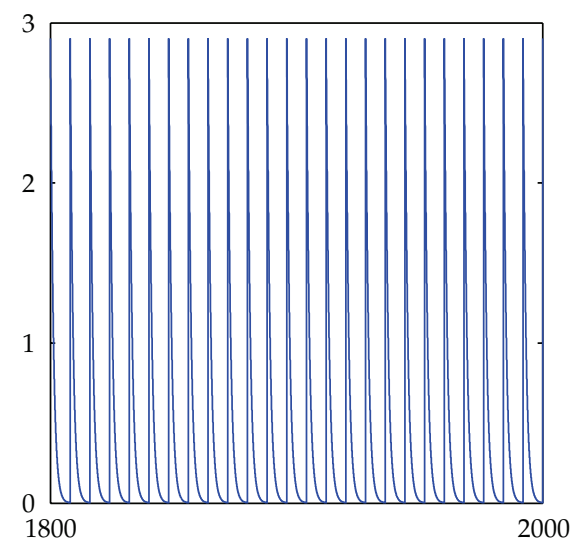

(c)

Figure 2: The dynamical behavior of the system (1.1) with parameters $a=5, T=8, \delta_{1}=0.2$. (a) (c) show that a trajectory with a starting point $\left(x_{0}, y_{0}, z_{0}\right)=(0.01,0.01,0.01)$ near the solution $\left(0,0, z^{*}(t)\right)$ is repelled from the periodic solution $\left(0,0, z^{*}(t)\right)$ and then approaches to another periodic solution.

Theorem 3.2. Suppose that aT $+\ln \left(1-\delta_{1}\right)>0$. Then the periodic solution $\left(x^{*}(t), 0, z^{*}(t)\right)$ is locally asymptotically stable if the condition

$$
\frac{\left(a+b \alpha_{1}\right)\left(c_{2}\left(A_{1}-A_{2}+A_{3}-A_{4}\right)-d_{1} d_{2} \beta_{2} T\right)+k_{1} c_{1} \beta_{2} d_{2} A_{5}}{\beta_{2} d_{2}\left(a+b \alpha_{1}\right)}<\ln \left(\frac{1}{1-\delta_{2}}\right)
$$

holds. Moreover, the periodic solution $\left(x^{*}(t), 0, z^{*}(t)\right)$ is globally asymptotically stable if the condition

$$
\frac{k_{1} c_{1} A_{5}}{a+b \alpha_{1}}-d_{1} T<\ln \left(\frac{1}{1-\delta_{2}}\right)
$$


holds. Here, the values $A_{i}$ are listed:

$$
\begin{aligned}
& A_{1}=\ln \left[\alpha_{2}\left(\exp \left(d_{2} T\right)-1+\delta_{3}\right)+\beta_{2} p \exp \left((1-l) d_{2} T\right)\right] \\
& A_{2}=\ln \left[\alpha_{2}\left(\exp \left(d_{2} T\right)-1+\delta_{3}\right)+\beta_{2} p \exp \left(d_{2} T\right)\right] \\
& A_{3}=\ln \left[\alpha_{2}\left(\exp \left(d_{2} T\right)-1+\delta_{3}\right)+\left(1-\delta_{3}\right) \beta_{2} p\right] \\
& A_{4}=\ln \left[\alpha_{2}\left(\exp \left(d_{2} T\right)-1+\delta_{3}\right)+\left(1-\delta_{3}\right) \beta_{2} p \exp \left((1-l) d_{2} T\right)\right] \\
& A_{5}=\ln \left[\frac{b \alpha_{1} \delta_{1} \exp (a T)+\left(\left(1-\delta_{1}\right) \exp (a T)-1\right)\left(a+b \alpha_{1}\right) \exp (a T)}{\left(a+b \alpha_{1}\right)(\exp (a T)-1)-a \delta_{1} \exp (a T)}\right] .
\end{aligned}
$$

The proof of this theorem is provided in Section 4. To illustrate an numerical example related to Theorem 3.2, let $b=0.5, c_{1}=2, c_{2}=2, \alpha_{1}=0.1, \alpha_{2}=0.5, \beta_{1}=1, \beta_{2}=0.1, d_{1}=$ $0.1, d_{2}=0.9, k_{1}=0.7, k_{2}=0.5, \delta_{2}=0.0001, \delta_{3}=0.03, l=0.6, p=3, a=5, T=8$, and $\delta_{1}=0.2$. These parameters satisfy the condition (3.1). It thus implies that trajectories asymptotically approach the periodic orbit $\left(x^{*}(t), 0, z^{*}(t)\right)$. In Figure 3, we numerically show that the periodic orbit $\left(x^{*}(t), 0, z^{*}(t)\right)$ is a sink.

\section{Proofs of Theorems 3.1 and 3.2}

\subsection{Proof of Theorem 3.1}

Proof. A local stability of the periodic solution $\left(0,0, z^{*}(t)\right)$ of the system (1.1) may be determined by considering the behavior of small amplitude perturbations of the solution. Let $(x(t), y(t), z(t))$ be any solution of the system (1.1). Define $x(t)=u(t), y(t)=v(t)$ and $z(t)=w(t)+z^{*}(t)$. Then they may be written as

$$
\left(\begin{array}{c}
u(t) \\
v(t) \\
w(t)
\end{array}\right)=\Phi(t)\left(\begin{array}{c}
u(0) \\
v(0) \\
w(0)
\end{array}\right)
$$

where $\Phi(t)$ satisfies

$$
\frac{d \Phi}{d t}=\left(\begin{array}{ccc}
a & 0 & 0 \\
0 & -d_{1}-\frac{c_{2} z^{*}(t)}{\beta_{2} z^{*}(t)+\alpha_{2}} & 0 \\
0 & \frac{k_{2} c_{2} z^{*}(t)}{\beta_{2} z^{*}(t)+\alpha_{2}} & -d_{2}
\end{array}\right) \Phi(t)
$$




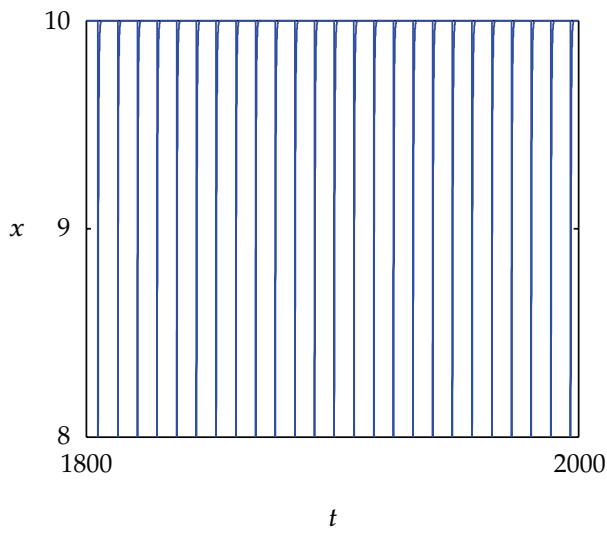

(a)

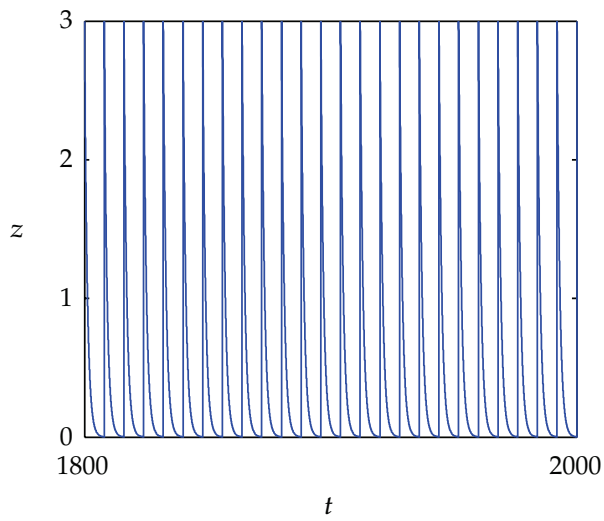

(c)

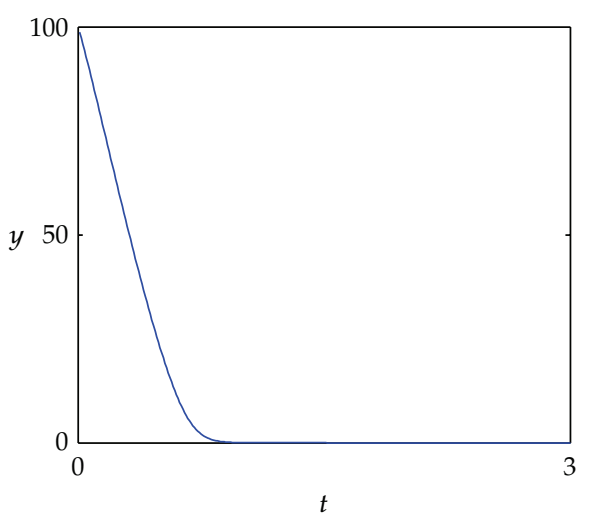

(e)

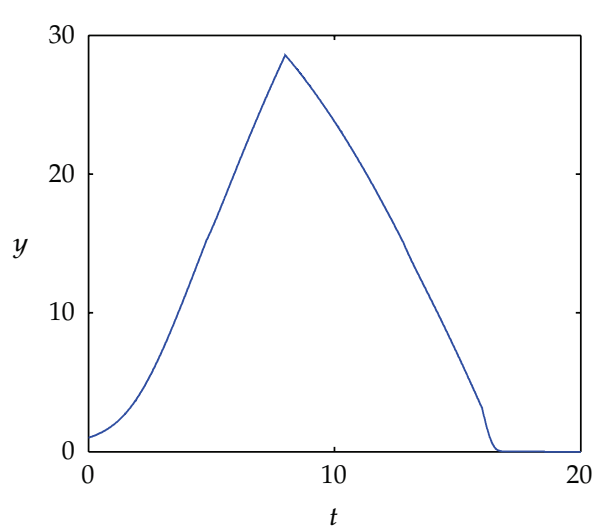

(b)

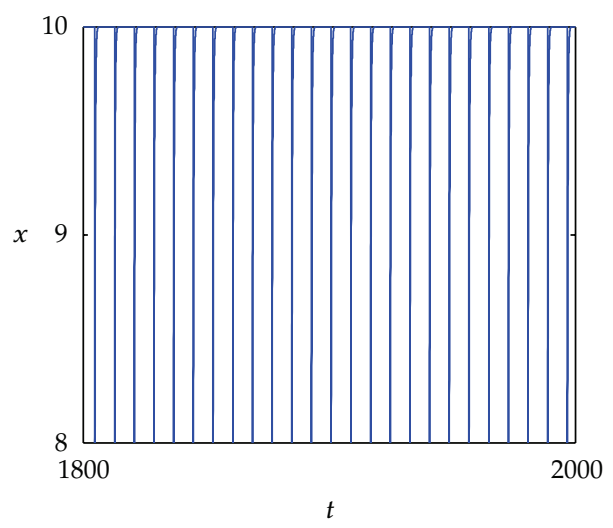

(d)

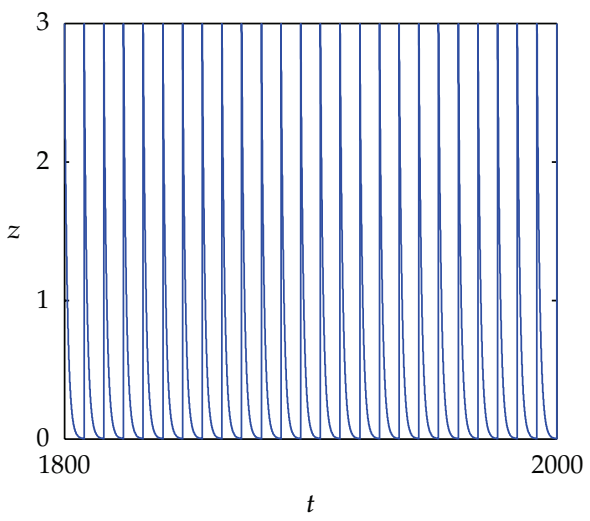

(f)

Figure 3: The dynamical behavior of the system (1.1). (a)-(c) show that a trajectory with a starting point $\left(x_{0}, y_{0}, z_{0}\right)=(10,1,0.4)$ approaches the periodic orbit $\left(x^{*}(t), 0, z^{*}(t)\right)$. In $(\mathrm{d})-(\mathrm{f})$, the behavior of trajectory with a different starting point $\left(x_{0}, y_{0}, z_{0}\right)=(100,100,100)$ is shown. 
and $\Phi(0)=I$ is the identity matrix. Therefore, the fundamental solution matrix is

$$
\Phi(t)=\left(\begin{array}{ccc}
\exp (a t) & 0 & 0 \\
0 & \exp \left(\int_{0}^{t}\left[-d_{1}-\frac{c_{2} z^{*}(s)}{\beta_{2} z^{*}(s)+\alpha_{2}}\right] d s\right) & 0 \\
0 & \exp \left(\int_{0}^{t} k_{2} c_{2} z^{*}(s) d s\right) & \exp \left(-d_{2} t\right)
\end{array}\right) .
$$

The resetting impulsive conditions of the system (1.1) become

$$
\begin{gathered}
\left(\begin{array}{c}
u\left((n+l-1) T^{+}\right) \\
v\left((n+l-1) T^{+}\right) \\
u\left((n+l-1) T^{+}\right)
\end{array}\right)=\left(\begin{array}{ccc}
1-\delta_{1} & 0 & 0 \\
0 & 1-\delta_{2} & 0 \\
0 & 0 & 1-\delta_{3}
\end{array}\right)\left(\begin{array}{c}
u((n+l-1) T) \\
v((n+l-1) T) \\
w((n+l-1) T)
\end{array}\right), \\
\left(\begin{array}{c}
u\left(n T^{+}\right) \\
v\left(n T^{+}\right) \\
w\left(n T^{+}\right)
\end{array}\right)=\left(\begin{array}{lll}
1 & 0 & 0 \\
0 & 1 & 0 \\
0 & 0 & 1
\end{array}\right)\left(\begin{array}{c}
u(n T) \\
v(n T) \\
w(n T)
\end{array}\right) .
\end{gathered}
$$

Note that the eigenvalues of

$$
S=\left(\begin{array}{ccc}
1-\delta_{1} & 0 & 0 \\
0 & 1-\delta_{2} & 0 \\
0 & 0 & 1-\delta_{3}
\end{array}\right)\left(\begin{array}{lll}
1 & 0 & 0 \\
0 & 1 & 0 \\
0 & 0 & 1
\end{array}\right) \Phi(T)
$$

are $\mu_{1}=\left(1-\delta_{1}\right) \exp (a T), \mu_{2}=\left(1-\delta_{2}\right) \exp \left(-\int_{0}^{T} d_{1}+\left(c_{2} z^{*}(s) /\left(\beta_{2} x^{*}(s)+\alpha_{2}\right)\right) d s\right)$ and $\mu_{3}=$ $\left(1-\delta_{3}\right) \exp \left(-d_{2} T\right)$. Clearly, $\mu_{2}<1$ and $\mu_{3}<1$. If $a T+\ln \left(1-\delta_{1}\right)>0$, then $\mu_{1}>1$. Therefore, by Floquet theory [24], the periodic solution $\left(0,0, z^{*}(t)\right)$ is unstable.

To prove a global stability of the periodic solution $\left(0,0, z^{*}(t)\right)$, first, we assume that $a T+\ln \left(1-\delta_{1}\right)<0$. Then $\mu_{1}=\left(1-\delta_{1}\right) \exp (a T)<1$. It means that the periodic solution $\left(0,0, z^{*}(t)\right)$ is locally asymptotically stable.

Let $(x(t), y(t), z(t))$ be any solution of (1.1). Take a number $\epsilon_{1}$ with $0<\epsilon_{1}<d_{1} \alpha_{1} / k_{1} c_{1}$ and let $\xi=\left(1-\delta_{1}\right) \exp \left(\left(-d_{1}+\left(k_{1} c_{1} / \alpha_{1}\right) \epsilon_{1}\right) T\right)$. Note that $0<\xi<1$. From the first equation in (1.1), we get

$$
x^{\prime}(t)=x(t)(a-b x(t))-\frac{c_{1} x(t) y(t)}{\alpha_{1}+x(t)+\beta_{1} y(t)} \leq x(t)(a-b x(t))
$$

for $t \neq(n+l-1) T$ and $t \neq n T$. By Lemma 2.2, we obtain $x(t) \leq \tilde{x}(t)$ for $t \geq 0$, where $\tilde{x}(t)$ is the solution of (2.4). Using Lemma 2.3, we also get that $\tilde{x}(t) \rightarrow 0$ as $t \rightarrow \infty$. It implies that there exists a number $T_{1}>0$ satisfying $x(t) \leq \epsilon_{1}$ for $t \geq T_{1}$. Without loss of generality, we 
may assume that $x(t) \leq \epsilon_{1}$ for all $t>0$. From the second equation in (1.1), we obtain that for $t \neq(n+l-1) T$ and $t \neq n T$,

$$
\begin{aligned}
y^{\prime}(t) & =-d_{1} y(t)+\frac{k_{1} c_{1} x(t) y(t)}{\alpha_{1}+x(t)+\beta_{1} y(t)}-\frac{c_{2} y(t) z(t)}{\alpha_{2}+y(t)+\beta_{2} z(t)} \\
& \leq-d_{1} y(t)+\frac{k_{1} c_{1}}{\alpha_{1}} x(t) y(t) \\
& \leq y(t)\left(-d_{1}+\frac{k_{1} c_{1}}{\alpha_{1}} \epsilon_{1}\right) .
\end{aligned}
$$

Integrating both sides of the inequality $(4.7)$ on $((n+l-1) T,(n+l) T]$, we get

$$
\begin{aligned}
y((n+l) T) & \leq y\left((n+l-1) T^{+}\right) \exp \left(\left(-d_{1}+\frac{k_{1} c_{1}}{\alpha_{1}} \epsilon_{1}\right) T\right) \\
& =y((n+l-1) T) \xi .
\end{aligned}
$$

It implies that $y((n+l) T) \leq y(l T) \xi^{n}$. Therefore $y((n+l) T) \rightarrow 0$ as $n \rightarrow \infty$. We also obtain that for $t \in((n+l-1) T,(n+l) T]$,

$$
\begin{aligned}
y(t) & \leq y\left((n+l-1) T^{+}\right) \exp \left(\left(-d_{1}+\frac{k_{1} c_{1}}{\alpha_{1}} \epsilon_{1}\right)(t-(n+l-1) T)\right) \\
& \leq y((n+l-1) T) .
\end{aligned}
$$

It thus implies that $y(t) \rightarrow 0$ as $t \rightarrow \infty$.

Now, take $0<\epsilon_{2}<\left(d_{2} \alpha_{2} / k_{2} c_{2}\right)$ in order to prove that $z(t) \rightarrow z^{*}(t)$ as $t \rightarrow \infty$. Since $\lim _{t \rightarrow \infty} y(t)=0$, there is a $T_{2}>0$ such that $y(t) \leq \epsilon_{2}$ for $t \geq T_{2}$. For the sake of simplicity, we assume that $y(t) \leq \epsilon_{2}$ for all $t \geq 0$. From the third equation in (1.1), we get that, for $t \neq(n+l-1) T$ and $t \neq n T$,

$$
\begin{aligned}
-d_{2} z(t) & \leq z^{\prime}(t)=-d_{2} z(t)+\frac{k_{2} c_{2} y(t) z(t)}{\alpha_{2}+y(t)+\beta_{2} z(t)} \\
& \leq\left(-d_{2}+\frac{k_{2} c_{2}}{\alpha_{2}} \epsilon_{2}\right) z(t) .
\end{aligned}
$$

Thus, by Lemma 2.2, we induce that $\widetilde{z}_{1}(t) \leq z(t) \leq \widetilde{z}_{2}(t)$, where $\widetilde{z}_{1}(t)$ is the solution of (2.6) and $\tilde{z}_{2}(t)$ is also the solution of (2.6) with $d_{2}$ changed into $d_{2}-\left(k_{2} c_{2} / \alpha_{2}\right) \epsilon_{2}$. Using Lemma 2.4 and letting $\epsilon_{2} \rightarrow 0, \tilde{z}_{1}(t)$ and $\tilde{z}_{2}(t)$ tend to $z^{*}(t)$ as $t \rightarrow \infty$. We thus prove that $\left|z(t)-z^{*}(t)\right| \rightarrow 0$ as $t \rightarrow \infty$. 


\subsection{Proof of Theorem 3.2}

To determine the stability of the periodic solution $\left(x^{*}(t), 0, z^{*}(t)\right)$, we will use the Floquet theory. First, we construct the monodromy matrix and calculate its eigenvalues:

$$
S=\left(\begin{array}{ccc}
1-\delta_{1} & 0 & 0 \\
0 & 1-\delta_{2} & 0 \\
0 & 0 & 1-\delta_{3}
\end{array}\right)\left(\begin{array}{lll}
1 & 0 & 0 \\
0 & 1 & 0 \\
0 & 0 & 1
\end{array}\right) \Phi(T)
$$

where $\Phi(t)$ satisfies

$$
\frac{d \Phi}{d t}=\left(\begin{array}{ccc}
a-2 b x^{*}(t) & -\frac{c_{1} x^{*}(t)}{\alpha_{1}+x^{*}(t)} & 0 \\
0 & -d_{1}+\frac{k_{1} c_{1} x^{*}(t)}{\alpha_{1}+x^{*}(t)}-\frac{c_{2} z^{*}(t)}{\alpha_{2}+\beta_{2} z^{*}(t)} & 0 \\
0 & \frac{k_{2} c_{2} z^{*}(t)}{\alpha_{2}+\beta_{2} z^{*}(t)} & -d_{2}
\end{array}\right) \Phi(t)
$$

and $\Phi(0)=I$ is the identity matrix. Then all eigenvalues of the matrix $S$ are

$$
\begin{aligned}
& \mu_{1}=\left(1-\delta_{1}\right) \exp \left(\int_{0}^{T} a-2 b x^{*}(t) d t\right) \\
& \mu_{2}=\left(1-\delta_{2}\right) \exp \left(\int_{0}^{T}-d_{1}+\frac{k_{1} c_{1} x^{*}(t)}{\alpha_{1}+x^{*}(t)}-\frac{c_{2} z^{*}(t)}{\alpha_{2}+\beta_{2} z^{*}(t)} d t\right) \\
& \mu_{3}=\left(1-\delta_{3}\right) \exp \left(-d_{2} T\right)<1 .
\end{aligned}
$$

Note that

$$
\begin{gathered}
\int_{0}^{T} x^{*}(t) d t=\frac{1}{b}\left(\ln \left(1-\delta_{1}\right)+a T\right), \\
\int_{0}^{T} \frac{x^{*}(t)}{\alpha_{1}+x^{*}(t)} d t=\frac{1}{a+b \alpha_{1}} \ln \left(\frac{b \alpha_{1}\left(1-\eta_{1}\right)+\eta_{1}\left(a+b \alpha_{1}\right) \exp (a T)}{a \eta_{1}+b \alpha_{1}}\right), \\
\int_{0}^{T} \frac{z^{*}(t)}{\alpha_{2}+\beta_{2} z^{*}(t)} d t=\frac{1}{\beta_{2} d_{2}} \ln \left(\frac{\left(\eta_{2}+\beta_{2} p\right)\left(\eta_{2}+\beta_{2} p\left(1-\delta_{3}\right) \exp \left(-d_{2} l T\right)\right)}{\left(\eta_{2}+\beta_{2} p \exp \left(-d_{2} l T\right)\right)\left(\eta_{2}+\beta_{2} p\left(1-\delta_{3}\right) \exp \left(-d_{2} T\right)\right)}\right),
\end{gathered}
$$

where $\eta_{1}=\left(\left(1-\delta_{1}\right) \exp (a T)-1\right) /(\exp (a T)-1)$ and $\eta_{2}=\alpha_{2}\left(1-\left(1-\delta_{3}\right) \exp \left(-d_{2} T\right)\right)$.

From (4.14) and $a T+\ln \left(1-\delta_{1}\right)>0$, we get that $\mu_{1}<1$ and $\mu_{2}$ is equivalent to (3.1) in the statement of Theorem 3.2. By the hypothesis of Theorem 3.2, we obtain $\mu_{2}<1$. Finally, based on the Floquet theory [24], we get that $\left(x^{*}(t), 0, z^{*}(t)\right)$ is locally asymptotically stable.

Suppose that $a T+\ln \left(1-\delta_{1}\right)>0$ and (3.2) hold. 
Let $(x(t), y(t), z(t))$ be any solution of (1.1). The condition (3.2) implies

$$
\mu_{2} \leq\left(1-\delta_{2}\right) \exp \left(\int_{0}^{T}-d_{1}+\frac{k_{1} c_{1} x^{*}(t)}{\alpha_{1}+x^{*}(t)} d t\right)<1
$$

Thus the periodic solution $\left(x^{*}(t), 0, z^{*}(t)\right)$ is locally asymptotically stable. Further, we can choose $\epsilon_{3}>0$ such that

$$
0<\zeta \equiv\left(1-\delta_{2}\right) \exp \left(\int_{0}^{T}-d_{1}+\frac{k_{1} c_{1}\left(x^{*}(t)+\epsilon_{3}\right)}{\alpha_{1}+x^{*}(t)+\epsilon_{3}} d t\right)<1
$$

As the proof of Theorem 3.1, by Lemma 2.2, we obtain $x(t) \leq \tilde{x}_{2}(t)$ for $t \geq 0$, where $\tilde{x}_{2}(t)$ is the solution of (2.4). It follows from Lemma 2.3 that there exists a $T_{3}>0$ such that $x(t) \leq x^{*}(t)+\epsilon_{3}$ for $t \geq T_{3}$. Without loss of generality, we may assume that $x(t) \leq x^{*}(t)+\epsilon_{3}$ for $t \geq 0$. From the second equation in (1.1), we get that for $t \neq(n+l-1) T$ and $t \neq n T$,

$$
\begin{aligned}
y^{\prime}(t) & =-d_{1} y(t)+\frac{k_{1} c_{1} x(t) y(t)}{\alpha_{1}+x(t)+\beta_{1} y(t)}-\frac{c_{2} y(t) z(t)}{\alpha_{2}+y(t)+\beta_{2} z(t)} \\
& \leq y(t)\left(-d_{1}+\frac{k_{1} c_{1}\left(x^{*}(t)+\epsilon_{3}\right)}{\alpha_{1}+x^{*}(t)+\epsilon_{3}}\right) .
\end{aligned}
$$

By integrating both sides of $(4.17)$ on $((n+l-1) T,(n+l) T]$, we obtain that

$$
\begin{aligned}
y((n+l) T) & \leq y((n+l-1) T+) \exp \left(\int_{(n+l-1) T}^{(n+l) T}-d_{1}+\frac{k_{1} c_{1}\left(x^{*}(t)+\epsilon_{3}\right)}{\alpha_{1}+x^{*}(t)+\epsilon_{3}} d t\right) \\
& =y((n+l-1) T) \zeta .
\end{aligned}
$$

It implies that $y((n+l) T) \leq y(l T) \zeta^{n}$. Finally, we get that $y((n+l) T) \rightarrow 0$ as $n \rightarrow \infty$.

From the inequality

$$
y^{\prime}(t) \leq \frac{k_{1} c_{1}}{\alpha_{1}}\left(x^{*}(t)+\epsilon_{3}\right) y(t) \leq \frac{k_{1} c_{1}}{\alpha_{1}}\left(\frac{a}{b} \exp (a T)+\epsilon_{3}\right) y(t)
$$

we get

$$
y(t) \leq y((n+l-1) T)\left(1-\delta_{2}\right) \exp \left(\frac{k_{1} c_{1}}{\alpha_{1}}\left[\frac{a}{b} \exp (a T)+\epsilon_{3}\right] T\right)
$$

for $t \in((n+l-1) T,(n+l) T]$. Consequently $y(t) \rightarrow 0$ as $t \rightarrow \infty$.

In order to show that $\left|x(t)-x^{*}(t)\right| \rightarrow 0$ as $t \rightarrow \infty$, we take $\epsilon_{4}$ such that $0<\epsilon_{4}<a \alpha_{1} / c_{1}$. Since $\lim _{t \rightarrow \infty} y(t)=0$, there exists a $T_{5}>0$ such that $y(t)<\epsilon_{4}$ for $t>T_{5}$. For the sake of 
simplicity, we may suppose that $y(t)<\epsilon_{4}$ for all $t \geq 0$. Therefore, for $t \neq(n+l-1) T$ and $t \neq n T$, we obtain

$$
\begin{aligned}
x^{\prime}(t) & =x(t)(a-b x(t))-\frac{c_{1} x(t) y(t)}{\alpha_{1}+x(t)+\beta_{1} y(t)} \\
& \geq x(t)\left(\left[a-\frac{c_{1} y(t)}{\alpha_{1}}\right]-b x(t)\right) \\
& \geq x(t)\left(\left[a-\frac{c_{1} \epsilon_{4}}{\alpha_{1}}\right]-b x(t)\right) .
\end{aligned}
$$

Thus, from Lemma 2.2, we obtain that $\tilde{x}_{1}(t) \leq x(t)$, where $\tilde{x}_{1}(t)$ is the solution of (2.4) with $a$ changed into $a-c_{1} \epsilon_{4} / \alpha_{1}$. From Lemma 2.3 and taking sufficiently small $\epsilon_{4}$, it is seen that $\tilde{x}_{1}(t)$ and $\tilde{x}_{2}(t)$ tend to $x^{*}(t)$ as $t \rightarrow \infty$. Thus, we get $\left|x(t)-x^{*}(t)\right| \rightarrow 0$ as $t \rightarrow \infty$.

Note that $-d_{2} z(t) \leq z^{\prime}(t)=-d_{2} z(t)+k_{2} c_{2} y(t) z(t) /\left(\alpha_{2}+y(t)+\beta_{2} z(t)\right) \leq-d_{2} z(t)+$ $\left(k_{2} c_{2} / \alpha_{2}\right) \epsilon_{4}$ for $t \neq(n+l-1) T$ and $t \neq n T$. By using the same process as the proof of Theorem 3.1, we can show that $\left|z(t)-z^{*}(t)\right| \rightarrow 0$ as $t \rightarrow \infty$.

\section{Conclusions}

We have considered the impulsively controlled three species food chain system with the Beddington-DeAngelis functional response proposed by the authors in [11]. To control the food chain system with three species, two control terms, biological and chemical controls, are employed. Here, a biological control means an impulsive and periodic releasing of toppredator with a fixed proportion and a chemical control means that, for instance, as a result of pesticide spreading fixed proportions of prey, mid-predator, and top-predator, their population will be impulsively degraded. Under controlling environment, we first show the conditions for extinction and growing of the prey and mid-predator using Floquet theory and comparison method. In addition, a suffcient condition for local and global stability of the mid-predator free solution is established, which means that if the mid-predator is regarded as the pest we can control the pest population under some conditions. These results will correct the misleading results shown in [11].

\section{Acknowledgments}

The first author was supported by the National Research Foundation of Korea (NRF) Grant funded by the Korea government (MEST) (no. R01-2008-000-20088-0) and the second author was supported by Basic Science Research Program through the National Research Foundation of Korea (NRF) funded by the Ministry of Education, Science, and Technology (20110006087).

\section{References}

[1] C. Cosner, D. L. Deangelis, J. S. Ault, and D. B. Olson, "Effects of spatial grouping on the functional response of predators," Theoretical Population Biology, vol. 56, no. 1, pp. 65-75, 1999.

[2] H. Baek, "Species extinction and permanence of an impulsively controlled two-prey one-predator system with seasonal effects," BioSystems, vol. 98, no. 1, pp. 7-18, 2009. 
[3] H. Baek and Y. Do, "Stability for a Holling type IV food chain system with impulsive perturbations," Kyungpook Mathematical Journal, vol. 48, no. 3, pp. 515-527, 2008.

[4] P. Georgescu and G. Morosanu, "Impulsive pertubations of a three-trophic prey-dependent food chain system," Mathematical and Computer Modelling, vol. 48, no. 7-8, pp. 975-997, 2008.

[5] Z. Li, W. Wang, and H. Wang, "The dynamics of a Beddington-type system with impulsive control strategy," Chaos, Solitons and Fractals, vol. 29, no. 5, pp. 1229-1239, 2006.

[6] B. Liu, Y. Zhang, and L. Chen, "Dynamic complexities in a Lotka-Volterra predator-prey model concerning impulsive control strategy," International Journal of Bifurcation and Chaos in Applied Sciences and Engineering, vol. 15, no. 2, pp. 517-531, 2005.

[7] B. Liu, Z. Teng, and L. Chen, "Analysis of a predator-prey model with Holling II functional response concerning impulsive control strategy," Journal of Computational and Applied Mathematics, vol. 193, no. 1, pp. 347-362, 2006.

[8] B. Liu, Y. J. Zhang, L. S. Chen, and L. H. Sun, "The dynamics of a prey-dependent consumption model concerning integrated pest management," Acta Mathematica Sinica, vol. 21, no. 3, pp. 541-554, 2005.

[9] X. Liu and L. Chen, "Complex dynamics of Holling type II Lotka-Volterra predator-prey system with impulsive perturbations on the predator," Chaos, Solitons and Fractals, vol. 16, no. 2, pp. 311-320, 2003.

[10] K. Negi and S. Gakkhar, "Dynamics in a Beddington-DeAngelis prey-predator system with impulsive harvesting," Ecological Modelling, vol. 206, no. 3-4, pp. 421-430, 2007.

[11] W. Wang, H. Wang, and Z. Li, "The dynamic complexity of a three-species Beddington-type food chain with impulsive control strategy," Chaos, Solitons and Fractals, vol. 32, no. 5, pp. 1772-1785, 2007.

[12] W. Wang, H. Wang, and Z. Li, "Chaotic behavior of a three-species Beddington-type system with impulsive perturbations," Chaos, Solitons and Fractals, vol. 37, no. 2, pp. 438-443, 2008.

[13] S. Zhang and L. Chen, "Chaos in three species food chain system with impulsive perturbations," Chaos, Solitons and Fractals, vol. 24, no. 1, pp. 73-83, 2005.

[14] S. Zhang and L. Chen, "A Holling II functional response food chain model with impulsive perturbations," Chaos, Solitons and Fractals, vol. 24, no. 5, pp. 1269-1278, 2005.

[15] S. Zhang and L. Chen, "A study of predator-prey models with the Beddington-DeAnglis functional response and impulsive effect," Chaos, Solitons and Fractals, vol. 27, no. 1, pp. 237-248, 2006.

[16] S. Zhang, F. Wang, and L. Chen, "A food chain model with impulsive perturbations and Holling IV functional response," Chaos, Solitons and Fractals, vol. 26, no. 3, pp. 855-866, 2005.

[17] S. Zhang, D. Tan, and L. Chen, "Dynamic complexities of a food chain model with impulsive perturbations and Beddington-DeAngelis functional response," Chaos, Solitons and Fractals, vol. 27, no. 3 , pp. 768-777, 2006.

[18] S. Zhang, D. Tan, and L. Chen, "Chaotic behavior of a periodically forced predator-prey system with Beddington-DeAngelis functional response and impulsive perturbations," Advances in Complex Systems, vol. 9, no. 3, pp. 209-222, 2006.

[19] Y. Zhang, B. Liu, and L. Chen, "Extinction and permanence of a two-prey one-predator system with impulsive effect," Mathematical Medicine and Biology, vol. 20, no. 4, pp. 309-325, 2003.

[20] J. Awrejcewicz, Bifurcation and Chaos in Simple Dynamical Systems, World Scientific, Singapore, 1989.

[21] J. Awrejcewicz, Bifurcation and Chaos in Coupled Oscillators, World Scientific, Singapore, 1991.

[22] J. Awrejcewicz and C.-H. Lamarque, Bifurcation and Chaos in Nonsmooth Mechanical Systems, vol. 45, World Scientific Publishing, Singapore, 2003.

[23] V. Lakshmikantham, D. D. Bainov, and P. S. Simeonov, Theory of Impulsive Differential Equations, World Scientific Publisher, Singapore, 1989.

[24] D. D. Bainov and P. S. Simeonov, "Impulsive differential equations: periodic solutions and applications," in Pitman Monographs and Surveys in Pure and Applied Mathematics, vol. 66, Longman Science \& Technical, Harlo, UK, 1993. 


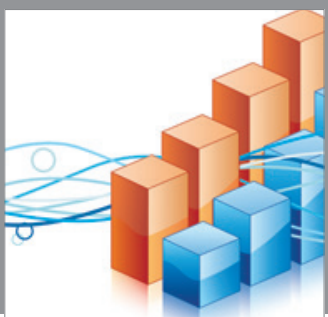

Advances in

Operations Research

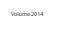

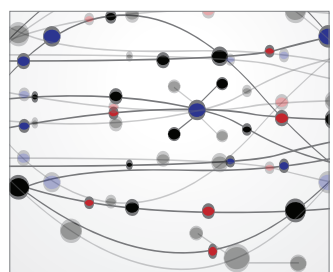

\section{The Scientific} World Journal
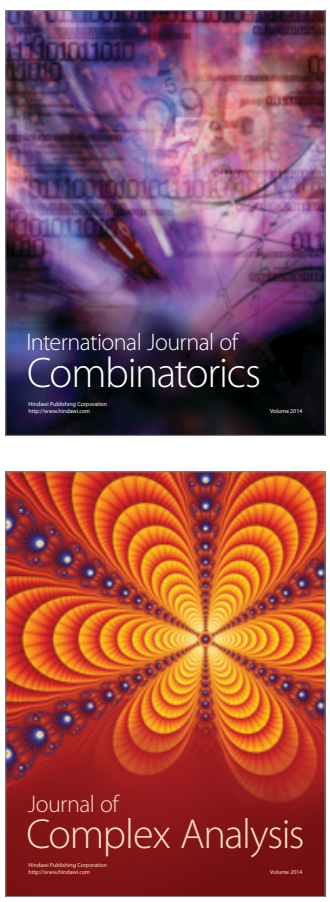

International Journal of

Mathematics and

Mathematical

Sciences
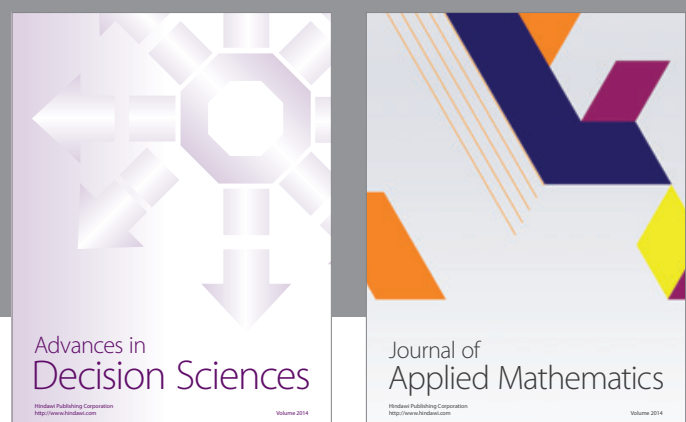

Journal of

Applied Mathematics
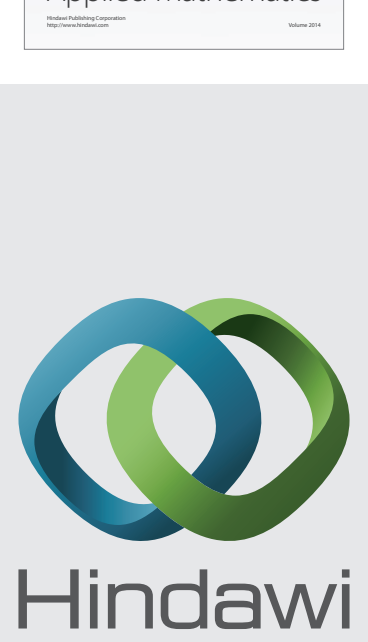

Submit your manuscripts at http://www.hindawi.com
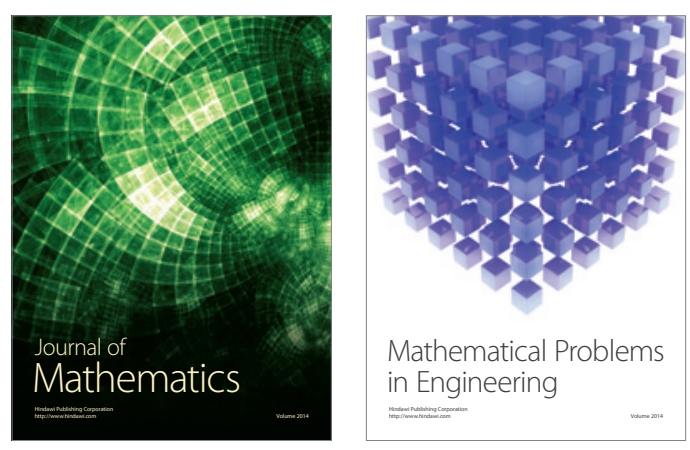

Mathematical Problems in Engineering
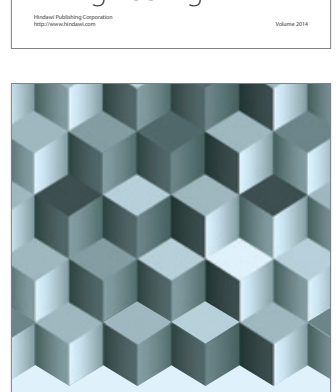

Journal of

Function Spaces
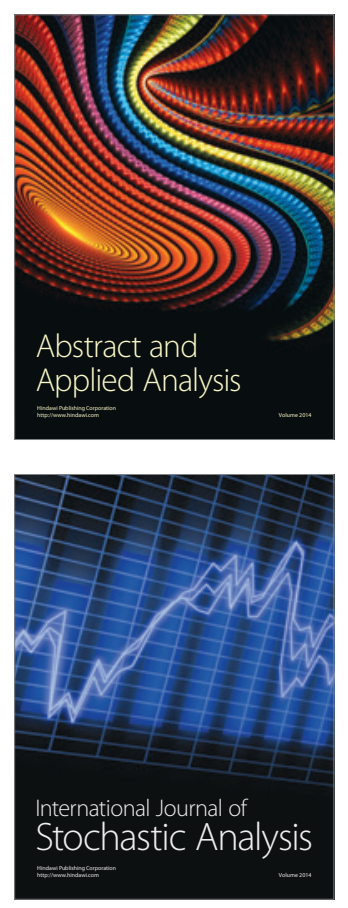

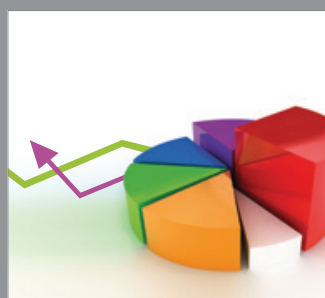

ournal of

Probability and Statistics

Promensencen
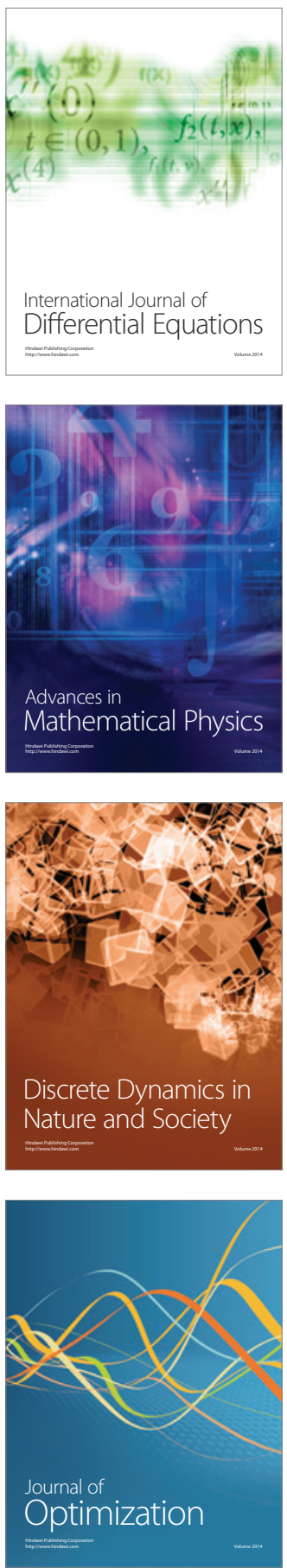